\title{
Ferroptosis and its facet in Cancer therapy
}

\begin{abstract}
Ferroptosis is a regulated process impelled by iron-dependent lipid peroxidation. It is a new type of cell destruction processes including apoptosis, autophagy and necrosis. It demonstrates mainly the contraction of mitochondria and expansion of mitochondrial membrane density which does not lead to any alteration in morphology. Due to the malfunctioning of ferroptosis several disorders arise which includes damage of one or more nerve which leads to numbness and muscle weakness whereas ischemia reperfusion injury, acute kidney failure and cancer also occurs. Also, ferroptosis is induced in large number of cancer cells through series of small molecules which helps in to bringing out this process. In scientific research and medicine many findings contribute in the chance of defeating cancer by genetic or pharmacological interference with ferroptosis cell death which is appealing for various researches. There are multiple pathways and cell organelles which plays a role in ferroptosis regulation. Ongoing studies on ferroptosis have demonstrated its role in humans though its mechanism is not yet clear. Recently, various studies have encouraged the role of this newly emerged cell death process and also showed some effective usage in the treatment of cancer. Here, we review the mitochondrial aspect of ferroptosis as well as discuss on the role of ferroptosis in Cancer cell therapy. We will also aim on the future scope of ferroptosis in the treatment of Cancer as well as discuss about the problems related to its clinical role which may trigger the cancer cell therapy.
\end{abstract}

Volume 6 Issue I - 202 I

\author{
Shreya Shreshtha,' Gopenath TS, ${ }^{2}$ Parthiban \\ $\mathrm{R},{ }^{3}$ Prathibha Rajashekara S, ${ }^{4}$ Kanthesh M \\ Basalingappa' \\ 'Division of Molecular Biology, School of Life Sciences, JSS \\ Academy of Higher Education and Research, Mysuru \\ 2Department of Biotechnology \& Bioinformatics, School of \\ Life Sciences, JSS Academy of Higher Education and Research, \\ Mysuru \\ ${ }^{3}$ Department of Clinical Laboratory Services \& Translational \\ Research, Malabar Cancer Centre, Kerala \\ ${ }^{4}$ Department of Botany, JSS College, Mysuru
}

Correspondence: Kanthesh M Basalingappa, M.Sc., Ph.D., FIAAM, Assistant Professor, Division of Molecular Biology, School of Life Sciences, JSS AHER, SS Nagara, Mysuru-5700 I5, Tel+9l 948204628I, Email kantheshmb@jssuni.edu.in

Received: January 05, 2021 | Published: January 19, 2021

Keywords: iron-dependent lipid peroxidation, necrosis, apoptosis, autophagy, mitochondrial membrane, cancer cells, multiple signalling pathways

\section{Introduction}

The term ferroptosis was first demonstrated by Dixon in $2012 .{ }^{1} \mathrm{He}$ described it as a novel cell death which was distinct from autophagy and apoptosis. Unlike other cell death, this is an iron and reactive oxygen species (ROS) dependent cell death and mostly, causes cytological changes. This includes shrunken mitochondria cristae, shredded outer mitochondrial membrane and membrane was compressed which causes change in the mitochondrial function. ${ }^{2-6}$ Due to the occurrence of oxidative stress and intense lipid peroxidation these deformities arise from the loss of selective permeability of plasma membrane. There are crucial regulators of ferroptosis which includes glutamate and glutamine. ${ }^{3}$ Ferroptosis occurs extracellularly along with typical physiological function. Sensitivity of cells to ferroptosis may affect the lipid metabolism. Ferroptosis also requires polyunsaturated fatty acids as they are susceptible to lipid peroxidation. ${ }^{4}$ Due to iron involution in the collection of lipid peroxide, ferroptosis occurs. ${ }^{5}$ Several molecules are involved in the modulation of ferroptosis are: voltage dependent anion channel (VDAC) $2 / 3$, glutathione peroxidase 4 (GPX4), heat shock protein $\beta-1$, nuclear factor E2-related factor 2 (NRF2), NADPH oxidase, the tumor suppressor p53 (TP53) and solute carrier family 7 member 1 (SLC7A1). ${ }^{6}$ It has explained in previous studies that ferroptosis played an important role in varied diseases including Cancer, ${ }^{7}$ acute kidney failure, ${ }^{8-10}$ neuropathy ${ }^{1,8}$ ischemia reperfusion injury. ${ }^{7}$

\section{Role of mitochondria}

As we know that, mitochondria have an important function in generating ATP through OXPHOS which is the basic requirement for most of the normal cell types. ${ }^{7,11}$ This mechanism is determined by the rate of ROS production. ${ }^{12}$ Mitochondria plays a main function in the tissue homeostasis and also causes crucifixion of several types of regulated cell death which includes extrinsic and intrinsic apoptosis and autophagy. ${ }^{13,14}$ In an experimental coronation of ferroptosis through pharmacological xCT inhibition demonstrated to bring out mitochondrial fragmentation, mitochondrial ROS production, loss of the mitochondrial membrane potential (MMP) and ATP depletion..$^{2,6,11,15-17}$ Therefore, there is a necessity of enhancing mitochondrial metabolism for crucifixion of ferroptosis, ${ }^{6}$ reduction of mitochondria via Parkin-mediated mitophagy in vitro or suppression of OXPHOS rescued cells from ferroptosis elicited by cystine deprivation or erastin. ${ }^{11}$ Thereby, due to oxidative stress and induction of ferroptosis causes mitochondrial DNA (mtDNA)-depleted $\rho 0$ cells to remain susceptible towards them. ${ }^{18}$ Therefore, it is still controversial that the role of mitochondria is involved in ferroptosis of all cell types or not and there may be cell-specific variations same as explained type I and type II cell events in extrinsic apoptosis. ${ }^{19}$ Meanwhile, $\mathrm{BH} 3$-interacting domain death agonist (BID) which is a Bcl-2 family member is split into shortened Bid (tBID) which has important role in extrinsic apoptosis type II cells and it was demonstrated for the necessity of erastin-induced ferroptosis and oxytosis in neurons. ${ }^{16}$

\section{Ferroptosis: overview in oncogenic selective cell death}

At first in small molecules particularly targeting Harvey rat sarcoma viral oncogene homolog (HRAS)G12V-mutant human foreskin fibroblasts (BJeLR) where ferroptosis was first originated as a component of synthetic fatality screen. ${ }^{20}$ Then two classes of small molecules were described, first class is described by Xc-inhibitor which has erastin and sulfasalazine and another class is described by GPX4 inhibition which has RSL3.,21 It was observed that due to increase in the intracellular iron levels of HRAS-mutant cells it was shown that HRASG12V selectivity was explained to stem from a higher expression of transferrin receptor TFRC. ${ }^{21}$ 
It was also demonstrated that the higher sensitivity to eras tin and the silencing of KRAS by small hairpin (sh) RNA leads to the reduction of eras tin's potency in Kirsten sarcoma viral oncogene homolog (KRAS)-mutant Calu-1 lung cancer cells. Also, in the treatment upon shRNA-mediated suppression of BRAF in A-673 cells which supresses an activating BRAFV600E mutation, showed more resistant to eras tin. ${ }^{2}$ Also, by increasing the activity of mitogenactivated protein kinase Pathway (MAPK), it was shown that human mammary epithelial (HME) cells expressing mutant epithelial growth factor receptor (EGFR) were much more sensitive to cystine deprivation-induced ferroptosis which encourages an increased ferroptosis sensitivity of oncogene-expressing cells. ${ }^{22}$ Additionally, it was presented that the level of MAPK pathway activity in nonsmall-cell lung cancer (NSCLC) cell lines, interacts with sensitivity to ferroptosis induced by cystine deprivation. ${ }^{22}$

Few Studies are also going on oncogenic RAS isoforms and introduction of cellular ROS aside from carry out the potential effects of ferroptosis. In this view, due to ROS production the oncogenic expression of NRASG12D and HRASG12V was and also causes a p38 mitogen-activated protein kinase (MAPK)-mediated oxidative stress response. ${ }^{23}$ Furthermore, the RAS-stimulated ROS production is mediated by PI3K/Rac1 and RAF/MEK/ERKRAS-effector pathways regulation which activates of nicotinamide adenine dinucleotide phosphate hydrogen (NADPH)-oxidases (NOX). ${ }^{24,25}$ KRAS also improved its activation and supported the cellular transformation by changing the location of $\mathrm{p} 47 \mathrm{phox}$, which is a sub- unit of NADPH oxidase 1 (NOX1) and shifting it to the plasma membrane. ${ }^{26}$ Also, in the background of arresting tumour suppressor p16 results in upregulation in NADHPH oxidase 4 due to KRASG12V expression. ${ }^{27}$ Thereby, oncogenic RAS isoforms may also have an impact in ferroptosis and lipid peroxidation by boosting up the delivery into the normal cellular ROS pool. This has been shown that due to the introduction of ROS belonging to acute oncogenic RAS over expression in vitro helps in cellular transformation at the rate of raised ROS. Still, there is a doubt arises by means of what the tumours expressing oncogenic KRAS from the remote site would manage ROS in vivo. An appealing context in respect NRF2, which acts as a transcription factor which also activates antioxidant defence genes shows that expression of KRASG12D, BRAFV619E and MYCERT2 oncogenes from their respective remote site induces nuclear NRF2. ${ }^{28,29}$

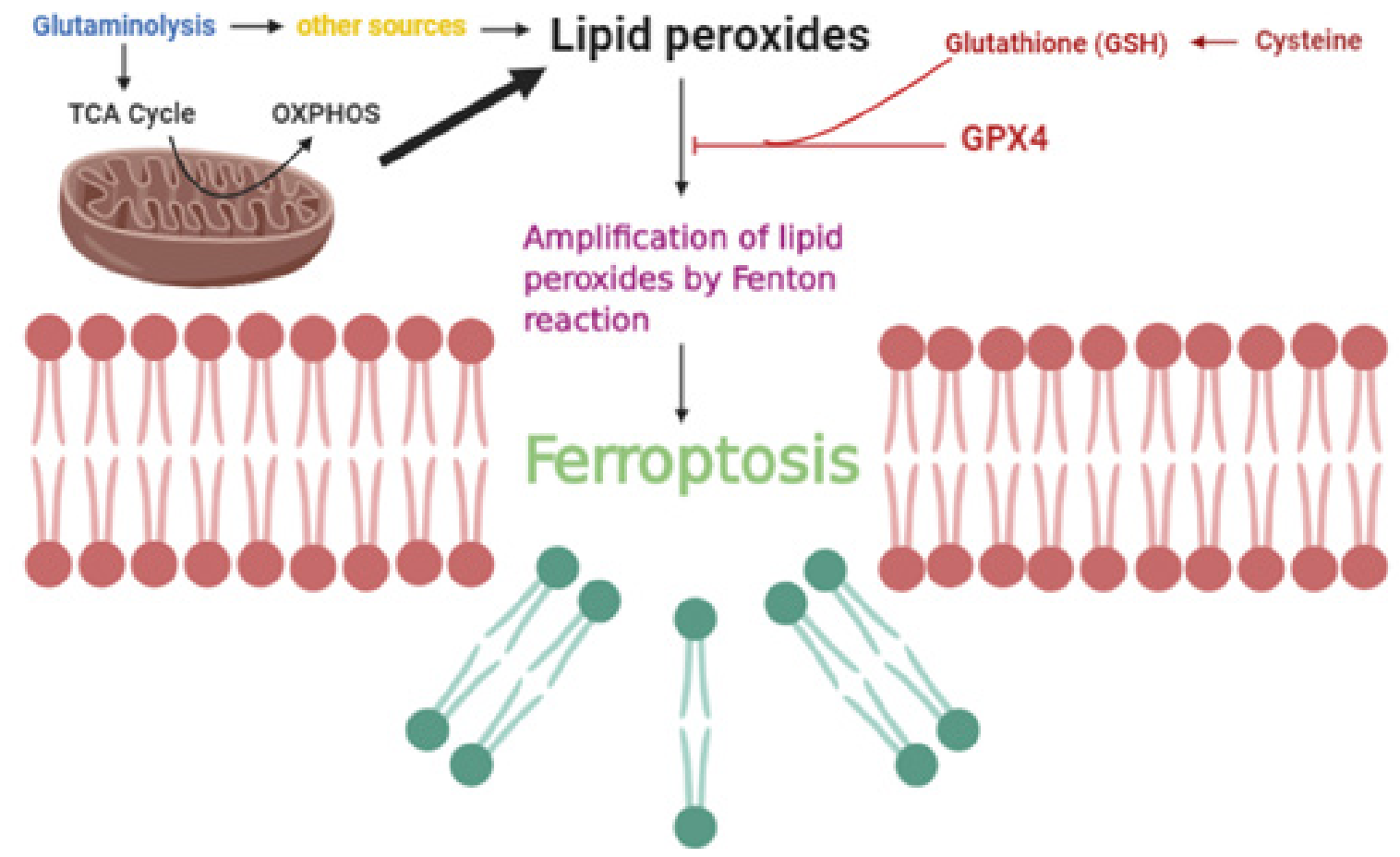

\section{Cysteine deprivation}

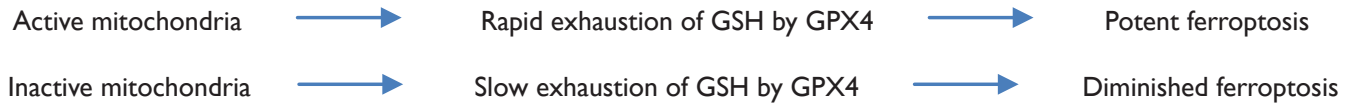

Figure I This shows that mitochondria play an essential role in cysteine-deprivation elicited ferroptosis but not in GPX4 inhibition elicited ferroptosis. The mitochondrial TCA cycle and electron transport chain encourage cysteine-deprivation elicited ferroptosis by delivering a main origin for cellular lipid peroxide production.Also, a component of the TCA namely fumarate hydratase may help in the anti-tumour role in mitochondrial mediated ferroptosis.

Mostly, NRF2 helps in the positive regulation of various genes namely glutamate-cysteine ligase catalytic subunit (GCLC) and glutamate-cysteine ligase modifier subunit (GCLM) and acts as a transcription factor during GSH (Figure 1) synthesis and also $\mathrm{xCT} .{ }^{30-32}$ Also, NRF2 enhances expression of Ferritin (FTH) ${ }^{33}$ which acts like a hunter for active iron thereby, improving its protecting function in ferroptosis. It was also seen that in chronic liver cancer, hepatocytes became more sensitive to ferroptosis inducers at the time of removal or inhibition of NRF2. ${ }^{33,34}$ Also, the expression of the ATP binding cassette (ABC)-family transporter which acts as a multidrug resistance protein 1(MRP1) is monitored by NRF2 when it merges with KEAP1 which is a tumour suppressor protein. 
Thereby, intervening glutathione efflux, MRP1 was able to sensitise to ferroptosis..$^{35}$ In human malignant lung carcinoma due to which cellular rates of glutamino lysis increases also, shows simultaneous elevation of KEAP1 and KRAS mutations. ${ }^{36}$ Also, activation of NRF2 by withaferin A resulting in the induction of its actual target gene heme oxygenase 1(HMOX1). In neuroblastoma, HMOX1 brings out a plenty of iron from cytosol by catalysing its release from heme promoting ferroptosis. ${ }^{37}$ Therefore, NRF2 activation results in cell type specific ferroptosis and expression of HMOX1 gene can also influence opposing results for ferroptosis.

\section{Cancer treatment and therapeutics}

Ferroptosis sensitivity of various types of carcinoma cells was significantly distinct from one other. In a committee of various cancer cell lines called NCI-60 of US National Cancer Institute Developmental Therapeutics Program. Among all the eight tissues, diffuse large B cell lymphomas and Renal cell carcinoma showed more susceptibility to erastin-induced ferroptosis than other six tissues namely: the breast, lung, colon, melanocytes, central nervous system, and ovary. ${ }^{6}$ There was controversy that the susceptibility of various cell lines to ferroptosis is distinct to each other due to the of the change in their essential metabolic state. In many studies, ferroptosis has been described as a major element in inhibiting cancer growth as well killing the carcinoma cells. Thereby, diagnosis is a better attempt than following same typical chemotherapy method. Therefore, summarization of all the achievable means of ferroptosis is an effective approach towards the treatment of various cancer types and also in clinical application.

Discovery of human epidermal growth factor receptor (HER1/ HER2) tyrosine kinase inhibitor Lapatinib which has a high mesenchymal state and it was also selectively sensitised to the interference of ferroptosis brings out the discovery of ferroptosisinducing therapy (FIT) which showed its effectiveness in various types of cancer treatment. ${ }^{38,39}$ For first time studies also showed that the cells which are free from other ways of killing but those are selectively sensitised to ferroptosis. Recently, immunotherapy is developed which demonstrated sensitization of tumour cell ferroptosis. In regard of XCT which is abnormally expressed in many cancers and inducing ferroptosis may be not much efficient for cancer. There are two major inducers namely RSL3 and erastin have poor solubility of water and it is metabolically instable which makes it inappropriate for the in vivo application so, it does not match up to pharmacokinetic standards. ${ }^{39,40,41}$ Many attempts were taken for in vivo application of erastin in order to avoid this obstacle and show erastin more relevant. One of the approaches was regarding triple-negative breast cancer (TNBC)cells which were treated with erastin which were wrapped in exosomes covered with folate to specifically target folate receptoroverexpressing TNBC. ${ }^{42}$ Another approach was shown anti-tumour activity of stable form of erastin called piperazine-coupled erastin in a xenograft model using human fibrosarcoma HT-1080 cells. ${ }^{18,40}$ Also, in a SU-DHL-6DLBCL xenograft model imidazole-ketone erastin, a metabolically stable variant of erastin has demonstrated to reduce tumour growth. ${ }^{41}$ Although for enhancing clinical application, small molecule backbones for RSL3 and erastin will have to be more advance. Also, there are many drugs which are certified by food and drug administration (FDA) determines its function by ferroptosis induction in various cancer types. One of the drugs is Sorafenib, which is a multi-kinase inhibitor approved by FDA and helps in the treatment of hypernephroma and also in liver cancer which is also called HCC. Thereby, Sorafenib is demonstrated to inhibit system xc-
.${ }^{43}$ Additionally, ferropstatin-1 and iron-chelators suppresses sorafenib by inducing cell death in HCC. ${ }^{44}$ Tumour suppressor retinoblastoma protein (RB1) suppresses ferroptosis induced by sorafenib treatment was also explained ${ }^{45}$ which indicates a potential biomarker for sorafenib treatment-induced ferroptosis.

Another drug called Sulfasalazine (SAS) which was an FDAapproved drug was speculated to act as an anti-inflammatory drug for the treatment of rheumatoid arthritis and inflammatory bowel diseases (Crohn, ulcerative colitis). ${ }^{46}$ The targets of SAS are arachidonat-5lipoxygenase (ALOX-5), ${ }^{47}$ cyclooxygenase2 (COX-2) ${ }^{48}$ and nuclear factor 'kappa-light-chain-enhancer' (NF-kB) which were described. ${ }^{49}$ It has also been explained that these inhibit the system xc- subunit $\mathrm{xCT}$ and adequately induce ferroptosis in non-Hodgkin lymphoma cells. ${ }^{46}$ Altretamine (hexamethyl melamine) which is also FDAapproved alkylating antineoplastic drug which helps in the treatment of ovarian cancer. ${ }^{50}$ It has also demonstrated that inhibition of GPX4 (Figure 2) helps in adequately destroying U-2932 DLBCL cells in vitro. ${ }^{51}$ Moreover, Statins namely cerivastatin and simvastatin, have been explained in inducing ferroptosis in the human fibro sarcoma cell line HT-1080 by blocking the mevalonate pathway and thus helps in reducing the synthesis of CoenzymeQ10. ${ }^{38}$ Hence, the induction of ferroptosis may determine the treatment efficiency and also there are various drugs which of several already certified as cancer drugs which may diminish the clinical development approach for the therapeutic induction of ferroptosis in human cancers. ${ }^{52,53}$

\section{Conclusion and perspective}

According to the studies, we have seen that ferroptosis is giving us a new and great advance in anti-tumor therapies as well as many encouraging findings have shown the efficiency of ferroptosis in achieving the goal. Currently, various experiments are focusing on the removal of cancer cells those are resistant in nature and for carrying out this appealing approach, ferroptotic cell death is playing a new and remarkable role. Importantly, it has been advised that to obtain a mesenchymal cell state (e.g., epithelial-mesenchymal transition(EMT) or cancer stem cells) meta static dissemination regulation and chemo resistance are helpful. ${ }^{54}$ Regarding targeted therapies, cancer cells having high-mesenchymal state emerged as an important method for both acquired and denovo resistance. ${ }^{55,56}$ Constant cancer cells which are approved for going out from formal cytotoxic cure via a torpid state tumor demonstrated an undistinguishable selective reliance on the GPX4 pathway which has a great therapeutic strategy. ${ }^{57,58}$ Hence, ferroptosis is studied as a possible therapeutic strategy which can also revert the resistant therapy in cancer strategy. Ferroptosis is a type which is more acknowledged than other cell programmed death which shows more immunogenicity. Few studies have explained that drug reposition of SASP and artesunate shows anti-tumor therapeutic effects by activating ferroptosis. . $^{59,60}$

Also, ferroptosis brings out a newly explained cell death and advances in the regression of drug-resistance and enhances the immune system of the host. Ongoing, researches on ferroptosis has an encouraging aspect in the cancer treatment. Still, ferroptosis did not achieve a formal recognition in clinical aspect due to the ramification of cancer cells including P53 and Ras-mutant. There is another question in the development of ferroptotic resistance which was shown in erastin treatment in Hela cells. Therefore, in future studies we need to find out the systemic responses and mechanism of interlink between distinct types of regulated cell death apart from ferroptosis sensitivity and resistance which would help us to achieve the complete possibility of cancer treatment by using FIT. 


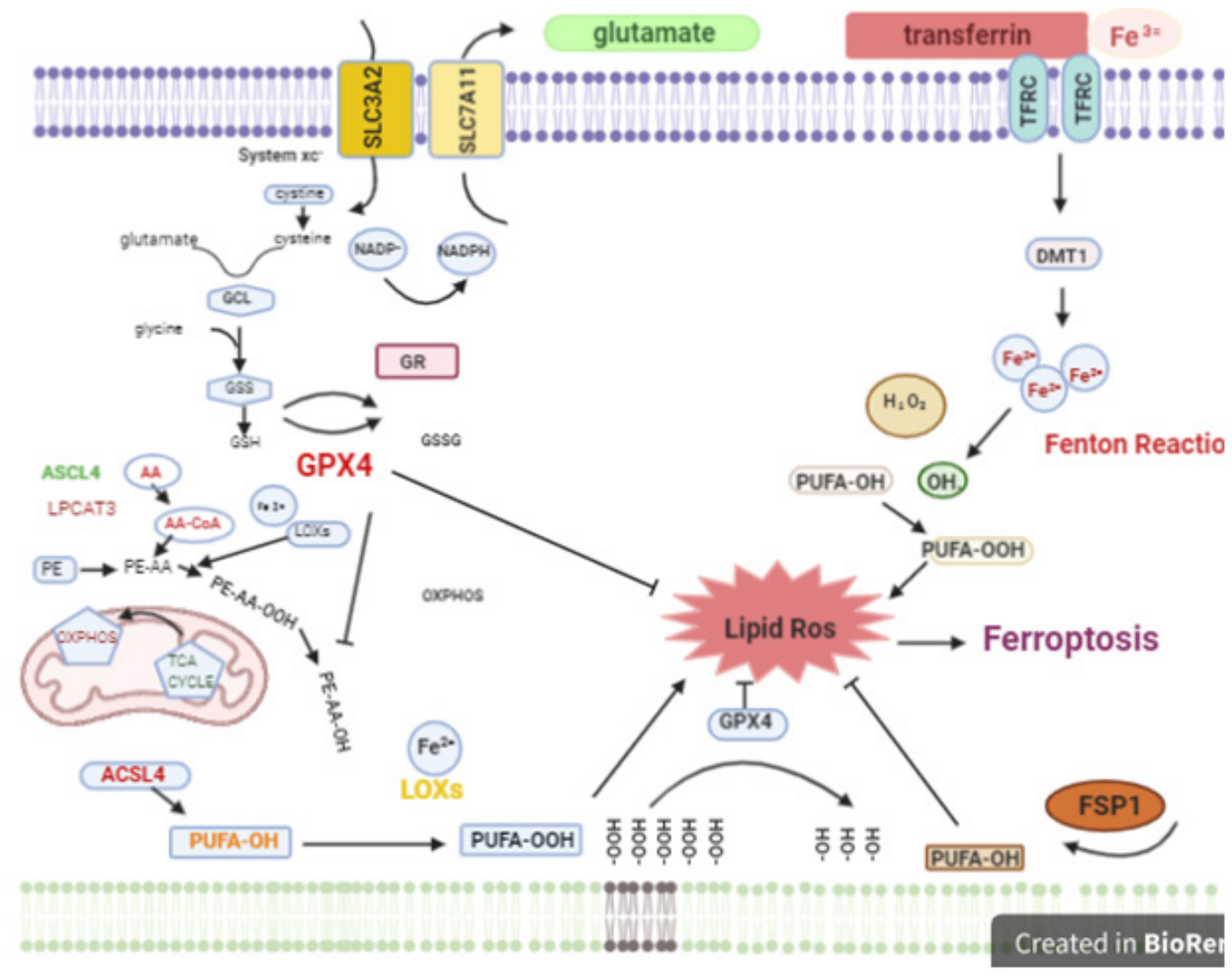

Figure 2 This is a pictorial representation of ferroptosis pathway. Ferroptosis follows abnormal progress of lipid reactive oxygen species (ROS) due to which the peroxidation $(-\mathrm{OOH})$ of polyunsaturated fatty acids (PUFAs) occur.The main peroxidation target PUFAs namely arachidonic acid (AA) phosphatidylethanolamine (PE) lipid species within cellular membranes results in membrane destruction and shredding. Lipid peroxidation carried out by cytosolic redox active iron $(\mathrm{Fe} 2+)$ moved into cells bound to transferrin via transferrin receptor (TFRC) endocytosis and endosomal release carry out by divalent metal transporter I (DMTI). Due to the existence of $\mathrm{H} 2 \mathrm{O} 2, \mathrm{Fe} 2+$ catalyses hydroxyl radical ( $\mathrm{HO}$ ) induce a Fenton reaction, which put in a radical lipid peroxidation chain reaction. Lipoxygenase (LOX) can also catalyse lipid peroxidation using Fe2+.As a required requirement for ferroptosis, Acyl-CoA synthetase long-chain family member 4 (ACSL4) and lysophosphatidyl choline acyltransferase 3 (LPCAT3) induce the pool of AA-containing target lipids. Glutathione peroxidase 4 (GPX4), successively, hydrolyses lipid peroxides by converting them into their respective non-toxic lipid alcohols (-OH). GPX4 needs glutathione (GSH) as a cofactor which upon its oxidation (GSSG) by GPX4 is reduced to GSH by glutathione reductase (GR). GSH synthesis depends on glutamate cysteine ligase (GCL) and glutathione synthetase (GSS) as well as on intracellular cystine shuttled into the cell in exchange for glutamate mediated by system xc-(SLC3A2 and SCL7AI I/ $\mathrm{xCT}$ ). Independently of GSH, ferroptosis suppressor protein I (FSPI) generates ubiquinol from ubiquinone which acts as a lipophilic radical trapping agent within membranes there by protecting from ferroptosis. Oxidative phosphorylation (OXPHOS) and the tricarboxylic acid (TCA)cycle have both been described to be needed for ferroptosis triggered by cystine-depletion or system xc-but not GPX4 inhibition.

\section{Author contributions}

Shreya S (SS) and Kanthesh BM (KBM) conceptualized the study. Shreya S (SS) and T. S. Gopenath (TSG) drafted the Manuscript. Parthiban R (PR), Prathibha Rajashekara S (PRS) and Kanthesh BM (KBM) helped with the Manuscript and Discussion.

\section{Acknowledgments}

The authors would like to acknowledge the Management of JSS Academy of Higher Education \& Research, Mysuru, Karnataka, for supporting the basic research ideas and also for the resources provided.

\section{Conflicts of interest}

The authors declare that there are no conflicts of interest.

\section{Funding}

None.

\section{References}

1. Dixon SJ. Ferroptosis: bug or feature?. Immunol Rev. 2017;277(1):150 157.

2. Yagoda N, von Rechenberg M, Zaganjor E, et al. RAS-RAF-MEKdependent oxidative cell death involving voltage-dependent anion channels. Nature. 2007;447(7146):865-869.

3. Yu H, Guo P, Xie X, et al. Ferroptosis, a new form of cell death, and its relationships with tumourous diseases. J Cell Mol Med. 2017;21(4):648657.

4. Latunde-Dada GO. Ferroptosis: role of lipid peroxidation, iron and ferritinophagy. Biochim Biophys Acta Gen Subj. 2017;1861(8):1893-900.

5. Stockwell BR, Angeli JP, Bayir H, et al. Ferroptosis: a regulated cell death nexus linking metabolism, redox biology, and disease. Cell;171(2):273285.

6. Xie Y, Hou W, Song X, et al. Ferroptosis: process and function. Cell Death Differ. 2016;23(3):369-379. 
7. Gao M, Monian P, Quadri N, et al. Glutaminolysis and transferrin regulate ferroptosis. Mol Cell. 2015;59(2):298-308.

8. Skouta R, Dixon SJ, Wang J, et al. Ferrostatins inhibit oxidative lipid damage and cell death in diverse disease models. $J$ Am Chem Soc. 2014;136(12):4551-4556.

9. Angeli JP, Schneider M, Proneth B, et al. Inactivation of the ferroptosis regulator Gpx4 triggers acute renal failure in mice. Nat Cell Biol. 2014;16(12):1180-1191.

10. Linkermann A, Skouta R, Himmerkus N, et al. Synchronized renal tubular cell death involves ferroptosis. Proc Natl Acad Sci USA. 2014;111(47):16836-16841.

11. Gao M, Yi J, Zhu J, et al. Role of mitochondria in ferroptosis. Mol Cell. 2019;73(2):354-363.

12. Area-Gomez E, Guardia-Laguarta C, Schon EA, et al. Mitochondria, OxPhos, and neurodegeneration: cells are not just running out of gas. $J$ Clin Invest. 2019;129(1):34-45.

13. Mattson, MP, Gleichmann M, Cheng A. Mitochondria in neuroplasticity and neurological disorders. Neuron. 2008;60(5):748-766.

14. Xie L, Shi F, Tan Z, et al. Mitochondrial network structure homeostasis and cell death. Cancer Sci. 2018;109(12):3686-3694.

15. Zhou B, Liu J, Kang R, et al. Ferroptosis is a type of autophagy-dependent cell death. Semin Cancer Biol. 2020;66:89-100.

16. Neitemeier S, Jelinek A, Laino V, et al. BID links ferroptosis to mitochondrial cell death pathways. Redox Biol. 2017;12:558-570.

17. Yuan H, Li X, Zhang X, et al. CISD1 inhibits ferroptosis by protection against mitochondrial lipid peroxidation. Biochem Biophys Res Commun 2016;478(2):838-844.

18. Dixon SJ, Lemberg KM, Lamprecht MR, et al. Ferroptosis: An IronDependent Form of Non-Apoptotic Cell Death. Cell. 2012;149(5):1060 1072.

19. Von Karstedt S, Montinaro A, Walczak H. Exploring the TRAILs less travelled: TRAIL in cancer biology and therapy. Nat Rev Cancer. 2017;17(6):352-366.

20. Dolma S, Lessnick SL, Hahn WC, et al. Identification of genotypeselective antitumor agents using synthetic lethal chemical screening in engineered human tumor cells. Cancer cell. 2003;3(3):285-296.

21. Yang WS, Stockwell BR. Synthetic lethal screening identifies compounds activating iron-dependent, nonapoptotic cell death in oncogenic-RASharboring cancer cells. Chem Biol. 2008;15(3):234-245.

22. Poursaitidis I, Wang X, Crighton T, et al. Oncogene-selective sensitivity to synchronous cell death following modulation of the amino acid nutrient cystine. Cell Rep. 2017;18(11):2547-2556.

23. Hole PS, Pearn L, Tonks AJ, et al. Ras-induced reactive oxygen species promote growth factor-independent proliferation in human CD34+ hematopoietic progenitor cells. Blood. 2010;115(6):1238-1246.

24. Cho HJ, Jeong HG, Lee JS, et al. Oncogenic H-ras enhances DNA repair through Ras/PI3K/Rac1 pathway in NIH3T3 cells: Evidence for association with reactive oxygen species. J Biol Chem. 2002;277(22): 19358-66.

25. Adachi Y, Shibai Y, Mitsushita J, et al. Oncogenic Ras upregulates NADPH oxidase 1 gene expression through MEK-ERK-dependent phosphorylation of GATA-6. Oncogene. 2008;27(36):4921-4932.

26. Park MT, Kim MJ, Suh Y, et al. Novel signaling axis for ROS generation during K-Ras-induced cellular transformation. Cell Death Differ. 2014;21(8):1185-1197.

27. Ju HQ, Ying H, Tian T, et al. Mutant Kras-and p16-regulated NOX4 activation overcomes metabolic checkpoints in development of pancreatic ductal adenocarcinoma. Nat Commun. 2017;8(1):1-4.
28. DeNicola GM, Karreth FA, Humpton TJ, et al. Oncogene-induced Nrf2 transcription promotes ROS detoxification and tumorigenesis. Nature. 2011;475(7354):106-109.

29. Nguyen T, Nioi P, Pickett CB. The Nrf2-antioxidant response element signaling pathway and its activation by oxidative stress. J Biol Chem. 2009;284(20):13291-13295.

30. Chen D, Tavana O, Chu B, et al. NRF2 is a major target of ARF in p53independent tumor suppression. Mol Cell. 2017;68(1):224-232.

31. Wakabayashi N, Itoh K, Wakabayashi J,et al. Keap1-null mutation leads to postnatal lethality due to constitutive Nrf2 activation. Nat Genet. 2003;35(3):238-245.

32. Ishii $\mathrm{T}$, Itoh $\mathrm{K}$, Takahashi $\mathrm{S}$, et al. Transcription factor Nrf2 coordinately regulates a group of oxidative stress-inducible genes in macrophages. $J$ Biol Chem. 2000;275(21):16023-16029.

33. Sun X, Ou Z, Chen R, et al. Activation of the p62-Keap1-NRF2 pathway protects against ferroptosis in hepatocellular carcinoma cells. Hepatology. 2016;63(1):173-184.

34. Arlt A, Sebens S, Krebs S, et al. Inhibition of the Nrf2 transcription factor by the alkaloid trigonelline renders pancreatic cancer cells more susceptible to apoptosis through decreased proteasomal gene expression and proteasome activity. Oncogene. 2013;32(40):4825-4835.

35. Cao JY, Poddar A, Magtanong L, et al. A genome-wide haploid genetic screen identifies regulators of glutathione abundance and ferroptosis sensitivity. Cell Rep. 2019;26(6):1544-1556.

36. Romero R, Sayin VI, Davidson SM, et al. Keap1 loss promotes Krasdriven lung cancer and results in dependence on glutaminolysis. Nat Med. 2017;23(11):1362-1368.

37. Hassannia B, Wiernicki B, Ingold I, et al. Nano-targeted induction of dual ferroptotic mechanisms eradicates high-risk neuroblastoma. J Clin Invest. 2018;128(8):3341-3355.

38. Viswanathan VS, Ryan MJ, Dhruv HD, et al. Dependency of a therapyresistant state of cancer cells on a lipid peroxidase pathway. Nature. 2017;547(7664):453-457.

39. Hangauer MJ, Viswanathan VS, Ryan MJ, et al. Drug-tolerant persister cancer cells are vulnerable to GPX4 inhibition. Nature. 2017;551(7679):247-250.

40. Yang WS, SriRamaratnam R, Welsch ME, et al. Regulation of Ferroptotic Cancer Cell Death by GPX4. Cell. 2014;156(1-2):317-331.

41. Zhang Y, Tan H, Daniels JD, et al. Imidazole Ketone Erastin Induces Ferroptosis and Slows Tumor Growth in a Mouse Lymphoma Model. Cell Chem Biol. 2019;26(5):623-633.

42. Yu M, Gai C, Li, Z, et al. Targeted exosomes-encapsulated erastin induced ferroptosis in the triple negative breast cancer cells. Cancer Sci.2019;110(10):3173-3182.

43. Dixon SJ, Patel DN, Welsch M, et al. Author response: Pharmacological inhibition of cystine-glutamate exchange induces endoplasmic reticulum stress and ferroptosis. Elife. 2014;3:e02523

44. Louandre, C, Ezzoukhry Z, Godin C, et al. Iron-dependent cell death of hepatocellular carcinoma cells exposed to sorafenib. Int $J$ Cancer. 2013;133(7):1732-1742.

45. Louandre C, Marcq I, Bouhlal H, et al. The retinoblastoma $(\mathrm{Rb})$ protein regulates ferroptosis induced by sorafenib inhuman hepatocellular carcinoma cells. Cancer Lett. 2015; 356(2 Pt B):971-917.

46. Gout PW, Buckley AR, Simms CR, et al. Sulfasalazine, a potent suppressor of lymphoma growth by inhibition of the $\mathrm{x}(\mathrm{c})$-cystine transporter: A new action for an old drug. Leukemia. 2001;15(10):1633-1640.

47. Nielsen OH, Bukhave K, Elmgreen J, et al. Inhibition of 5-lipoxygenase pathway of arachidonic acid metabolism in human neutrophils by sulfasalazine and 5-aminosalicylic acid. Dig Dis Sci. 1987;32(6):577582. 
48. Pruzanski W, Stefanski E, Vadas Pet al. Inhibition of extracellula release of pro inflammatory secretory phospholipase A2 (sPLA2) by sulfasalazine: A novel mechanism of anti-inflammatory activity. Biochem Pharmacol.1997;53(12):1901-1907.

49. Weber CK, Liptay S, Wirth T, et al. Suppression of NF-kappaB activity by sulfasalazine is mediated by direct inhibition of I kappa B kinases alpha and beta. Gastroenterology. 2000;119(5):1209-1218.

50. Damia G, D'lncalci M. Clinical Pharmacokinetics of Altretamine. Clin Pharmacokinet.1995;28(6):439-448.

51. Woo JH, Shimoni Y, Yang WS, et al. Elucidating Compound Mechanism of Action by Network Perturbation Analysis. Cell. 2015;162(2):441-451.

52. Hassannia B, Vandenabeele P, Vanden Berghe, T. Targeting Ferroptosis to Iron Out Cancer. Cancer Cell. 2019;35(6):830-849.

53. Florean C, Song S, Dicato M, et al. Redox biology of regulated cell death in cancer: A focus on necroptosis and ferroptosis. Free Radic Biol Med. 2019;134:177-189.

54. Pattabiraman DR, Weinberg RA. Tackling the cancer stem cells-what challenges do they pose? Nat Rev Drug Discov. 2014;13(7):497-512.
55. Salt MB, Bandyopadhyay S, McCormick F. Epithelial-to-mesenchymal transition rewires the molecular path to PI3K-dependent proliferation. Cancer Discovery. 2014; 4:186-199.

56. Rizos H, Menzies AM, Pupo GM, et al. BRAF inhibitor resistance mechanisms in metastatic melanoma: spectrum and clinical impact. Clin Cancer Res. 2014;20(7):1965-1967.

57. Galluzzi L, Vitale I, Aaronson SA, et al. Molecular mechanisms of cell death: recommendations of the Nomenclature Committee on Cell Death 2018. Cell Death Differ. 2018;25(3):486-541.

58. Ahern TP, Lash TL, Damkier P, et al. Statins and breast cancer prognosis: evidence and opportunities. Lancet Oncol. 2014;15(10):e461-e468.

59. Yoshida GJ. Therapeutic strategies of drug repositioning targeting autophagy to induce cancer cell death: from pathophysiology to treatment. J Hematol Oncol. 2017;10(7):67.

60. Nie J, Lin B, Zhou M, et al. Role of ferroptosis in hepatocellular carcinoma. J Cancer Res Clin Oncol. 2018;144(12):2329-2337. 